INSTITUT NATIONAL DE RECHERCHE EN INFORMATIQUE ET EN AUTOMATIQUE

\title{
Numerical treatment of defective boundary conditions for the Navier-Stokes equations
}

Luca Formaggia — Jean-Frédéric Gerbeau — Fabio Nobile — Alfio Quarteroni

$\mathbf{N}^{\circ} 4093$

Janvier 2001

THÈME 4 



\title{
Numerical treatment of defective boundary conditions for the Navier-Stokes equations
}

\author{
Luca Formaggia $*^{*}$, Jean-Frédéric Gerbeau ${ }^{\dagger}$, Fabio Nobile ${ }^{*}$, Alfio Quarteroni ${ }^{\ddagger *}$ \\ Thème 4 - Simulation et optimisation \\ de systèmes complexes \\ Projet M3N
}

Rapport de recherche $n^{\circ} 4093$ - Janvier 2001 - 24 pages

\begin{abstract}
We present a formulation for accommodating defective boundary conditions for the incompressible Navier-Stokes equations where only averaged values are prescribed on measurable portion of the boundary. In particular we consider the case where the average mass flux is imposed on several domain sections. This methodology has an interesting application in the numerical simulation of flow in blood vessels, when only a reduced set of boundary data are generally available for the upstream and downstream sections.
\end{abstract}

Key-words: Navier-Stokes equations, flux boundary conditions, pressure boundary conditions, Lagrange multipliers

* Département de Mathématiques, École Polytechnique Fédérale, CH-1015, Lausanne, SWITZERLAND, email: Luca.Formaggia@epfl.ch, Fabio.Nobile@epfl.ch, Alfio.Quarteroni@epfl.ch

$\dagger$ INRIA Rocquencourt - Projet M3N - email: Jean-Frederic.Gerbeau@inria.fr

$\ddagger$ Dipartimento di Matematica "F. Brioschi", Politecnico di Milano, Piazza Leonardo da Vinci 32, I-20133 Milano, ITALY 


\section{Traitement numérique de conditions aux limites défectives pour les équations de Navier-Stokes}

Résumé : On présente une formulation des équations de Navier-Stokes avec des conditions aux limites portant sur des quantités moyennes. En particulier, on considère le cas où on impose le flux de masse sur plusieurs portions de la frontière du domaine. Cette méthodologie trouve une application dans la simulation numérique des écoulements sanguins, où seules des quantités moyennes sont généralement disponibles en entrée et en sortie du domaine de calcul.

Mots-clés : équations de Navier-Stokes, conditions aux limites sur le flux, conditions aux limites sur la pression, multiplicateurs de Lagrange 


\section{Introduction}

A necessary condition for the existence of the solution of the incompressible Navier-Stokes equations on a bounded domain $\Omega$ is that an appropriate set of boundary conditions is imposed on $\partial \Omega$. In a classical setting, at each point on the boundary one needs a number of conditions equal to the spatial dimension of the problem. Typically, one can prescribe the components of the velocity (Dirichlet boundary condition) or those of the Cauchy normal stress (Neumann boundary condition), or an appropriate combination of velocity and normal stress.

In this work, we will consider the rather different situation occurring when one has at disposal only a limited data set of boundary conditions, a-priori insufficient to "close" the differential problem at hand. We will refer to this incomplete set as defective boundary conditions. An example where this situation occurs is the simulation of blood flow in an artery, where measurements are normally able to provide only averaged velocity or pressure data at the upstream and downstream sections, and not complete fields as it would be required for the numerical computations. A similar situation occurs when one wants to simulate the cardiovascular system by multiscale approaches such those proposed in [5] and [3]. In that case, the boundary data for the Navier-Stokes equations do not come from real measurement but rather as the output of simplified models of the global cardiovascular system. These simple models, which are typically based on the solution of either a system of ordinary differential equations or of one-dimensional differential problems, normally provide the evolution of mean pressure and velocity inside the various regions of the cardiovascular system. If we want to use them to feed boundary data to a more detailed local model based on the solution of the Navier-Stokes equations, we need to have a way to "translate" these mean quantities in mathematically sound boundary conditions for the Navier-Stokes problem. This issue has been addressed in the cited references. An other interesting application of the technique is in the simulation of a free interface problem, when one wants to assure that the numerical approximation satisfies mass conservation. An example of a problem of this type is presented in the section dedicated to numerical experiments.

A possible approach is provided by the so called do-nothing boundary conditions proposed in [7]. In this paper we analyse another, somehow more flexible, alternative based on the use of Lagrange multipliers.

In the first section we address the problem in general terms and we introduce the functional setting for the analysis. We also give an overview of the do-nothing approach applied to problems where either an average mass flux or an average normal stress is imposed on a portion of the computational domain boundary. In Section 2 we introduce the alternative formulation given by a Lagrange multiplier approach and carry out its analysis. In Section 3 we propose several solution algorithms that are suitable for its implementation in the context of the solution of the NavierStokes equations by algebraic fractional step techniques. Finally, section 4 presents numerical results illustrating the effectiveness of the proposed methodology.

\section{Problem formulation and defective boundary conditions}

Let $\Omega$ be a bounded domain of $\mathbb{R}^{d}, d=2$ or 3 , whose boundary $\partial \Omega$ is decomposed into the union of $\Gamma$ and several disjoint sections $S_{0}, S_{1}, \ldots, S_{n}, n \geq 1$ (see Figure 1).

We are interested in solving the Navier-Stokes equations in $\Omega$

$$
\left\{\begin{aligned}
\partial_{t} \mathbf{u}+\mathbf{u} \cdot \nabla \mathbf{u}+\nabla p-\nu \Delta \mathbf{u} & =\mathbf{f}, & t>0 \\
\operatorname{div} \mathbf{u} & =0, & t>0 \\
\mathbf{u} & =\mathbf{u}_{0}, & t=0,
\end{aligned}\right.
$$

supplemented by homogeneous boundary conditions on $\Gamma$,

$$
\left.\mathbf{u}\right|_{\Gamma}=0,
$$

while two different kinds of boundary conditions will be considered on the sections $S_{i}, i=0, \ldots, n$. Both are well suited for blood flow simulations $[12,3]$, where $\Omega$ would represent the portion of an 


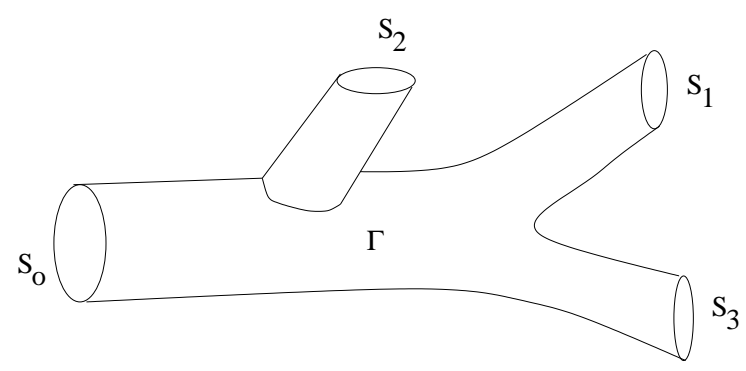

Figure 1: The partition of the boundary of the domain $\Omega$.

artery, $\Gamma$ the vessel wall and $S_{i}$ the artificial upstream and downstream sections. Even if for this specific problem the vessel wall should be considered moving with time because of the flexibility of the vessel wall structure, here we will address only the case where $\Gamma$ is fixed.

The first condition we consider is the so-called prescribed mean pressure problem which requires that

$$
\frac{1}{\operatorname{meas}\left(S_{i}\right)} \int_{S_{i}} p d s=P_{i}, \quad i=0, \ldots, n,
$$

where each $P_{i}$ is a prescribed function of the time $t$, constant on $S_{i}$. The second condition we wish to address is the prescribed flux problem

$$
\int_{S_{i}} \mathbf{u} \cdot \mathbf{n} d s=Q_{i}, \text { for } i=0, \ldots, n,
$$

where the $Q_{i}$ 's are assigned functions of time. Due to the incompressibility of the fluid, a compatibility relation must exist among the fluxes $Q_{i}$, namely $Q_{0}$ must be equal to $-\sum_{i=1}^{n} Q_{i}$.

The initial-boundary value problem (1)-(2) with either (3) or (4) is not well-posed from a physical point of view, since its solution is not unique. Indeed, on every section $S_{i}$, we are prescribing just one scalar condition rather than $d$ conditions at every point $\mathbf{x} \in S_{i}$, as it should be.

In [7] the do-nothing approach was advocated as a way of solving the two situations just presented. By this technique, a particular weak formulation is devised which allows to fulfill conditions (3) (resp. (4)) at some extent, giving rise to a well-posed problem. In fact, this formulation contains also "implicit" (Neumann-like) boundary conditions which select one particular solution among all the physical solutions of the original differential problem.

We will here give a brief presentation of this approach. Let us introduce the functional spaces

$$
V=\left\{\mathbf{v} \in\left[H^{1}(\Omega)\right]^{d},\left.\mathbf{v}\right|_{\Gamma}=0\right\} \quad \text { and } \quad M=L^{2}(\Omega) .
$$

We suppose that $\mathbf{f} \in V^{\prime}$ and we introduce the functional $\phi_{i} \in V^{\prime}, i=0, \ldots, n$ which measures the flux of a vector function through the surface $S_{i}$. Precisely

$$
<\phi_{i}, \mathbf{v}>=\int_{S_{i}} \mathbf{v} \cdot \mathbf{n} d s, \quad \forall \mathbf{v} \in V,
$$

where $\mathbf{n}$ is the outward unit normal vector on $\partial \Omega$. For this reason $\phi_{i}$ is called the flux functional on $S_{i}$.

Then, the "do-nothing" formulation for the pressure drop problem reads : find $\mathbf{u} \in V$ and $p \in M$ such that, for all $\mathbf{v} \in V$ and $q \in M$,

$$
\left\{\begin{aligned}
\left(\partial_{t} \mathbf{u}+\mathbf{u} \cdot \nabla \mathbf{u}, \mathbf{v}\right)+\nu(\nabla \mathbf{u}, \nabla \mathbf{v})-(p, \operatorname{div} \mathbf{v}) & =<\mathbf{f}, \mathbf{v}>-\sum_{i=0}^{n} P_{i}\left\langle\phi_{i}, \mathbf{v}\right\rangle \\
(q, \operatorname{div} \mathbf{u}) & =0
\end{aligned}\right.
$$


for all $t>0$, with $\mathbf{u}=\mathbf{u}_{0}$ for $t=0$.

It follows easily, by using the Green formula, that the solution of (5) satisfies

$$
\left.\left(p-\nu \frac{\partial u_{n}}{\partial \mathbf{n}}\right)\right|_{S_{i}}=P_{i},\left.\quad \frac{\partial \mathbf{u}_{\tau}}{\partial \mathbf{n}}\right|_{S_{i}}=0, \quad \text { for } i=0, \ldots, n,
$$

where we have set $u_{n}=\mathbf{u} \cdot \mathbf{n}$ and $\mathbf{u}_{\tau}=\mathbf{u}-u_{n} \mathbf{n}$.

Thus

$$
\frac{1}{\operatorname{meas}\left(S_{i}\right)} \int_{S_{i}} p d s=P_{i}+\frac{\nu}{\operatorname{meas}\left(S_{i}\right)} \int_{S_{i}} \frac{\partial u_{n}}{\partial \mathbf{n}} d s .
$$

We conclude that the desired condition (3) is recovered exactly only in those cases where the last integral in (6) vanishes. This occurs, for instance, when $S_{i}$ is a plane section perpendicular to a cylindrical pipe. Otherwise, $P_{i}$ will be the mean value of the normal component of the normal stresses on $S_{i}$.

For the prescribed flux problem, the "do-nothing" approach is formulated as follows. Let us introduce the space

$$
V^{\star}=\left\{\mathbf{v} \in V,<\phi_{i}, \mathbf{v}>=0, i=0, \ldots, n\right\},
$$

and the vector functions $\mathbf{b}_{i} \in V, \quad i=1, \ldots, n$ (called flux-carriers) that satisfy:

$$
\operatorname{div} \mathbf{b}_{i}=0, \quad \int_{S_{0}} \mathbf{b}_{i} \cdot \mathbf{n} d s=-1, \quad \int_{S_{j}} \mathbf{b}_{i} \cdot \mathbf{n} d s=\delta_{i j}, \quad \text { for } i, j=1, \ldots, n .
$$

The weak formulation of problem (1), (2), (4) proposed in [7] reads:

find $\mathbf{u}=\mathbf{w}+\sum_{i=1}^{n} Q_{i} \mathbf{b}_{i}$, with $\mathbf{w} \in V^{\star}$ and $p \in M \backslash \mathbb{R}$ such that for all $\mathbf{v} \in V^{\star}$ and $q \in M$

$$
\left\{\begin{aligned}
\left(\partial_{t} \mathbf{u}+\mathbf{u} \cdot \nabla \mathbf{u}, \mathbf{v}\right)+\nu(\nabla \mathbf{u}, \nabla \mathbf{v})-(p, \operatorname{div} \mathbf{v}) & =0 \\
(q, \operatorname{div} \mathbf{u}) & =0,
\end{aligned}\right.
$$

for all $t>0$, with $\mathbf{u}=\mathbf{u}_{0}$ for $t=0$.

The corresponding solution satisfies:

$$
\left.\left(p-\nu \frac{\partial u_{n}}{\partial \mathbf{n}}\right)\right|_{S_{i}}=P_{i},\left.\quad \frac{\partial \mathbf{u}_{\tau}}{\partial \mathbf{n}}\right|_{S_{i}}=0, \quad \text { for } i=0, \ldots, n,
$$

where the $P_{i}$ 's are a priori unknown constants (in space).

The formulation of the prescribed pressure drop problem may be easily discretised as it can be regarded as a classical Navier-Stokes problem with Neumann boundary conditions. On the other hand, the definition of the functional space $V^{\star}$ makes the implementation of the prescribed flux problem less straightforward.

\section{A Lagrange multipliers approach for flux boundary condi- tions}

In this section, we propose a slightly different formulation of the prescribed flux problem presented above. We consider (1) and (2) and we prescribe the flux on all but one section of $\partial \Omega$. More precisely, we aim at satisfying

$$
<\phi_{i}, \mathbf{u}>=\int_{S_{i}} \mathbf{u} \cdot \mathbf{n} d s=Q_{i}, \quad \text { for } i=1, \ldots, n,
$$

plus the following homogeneous Neumann boundary condition on $S_{0}$

$$
\left.\left(-p \mathbf{n}+\frac{\partial \mathbf{u}}{\partial \mathbf{n}}\right)\right|_{S_{0}}=0
$$


The motivation of such an approach will be clarified in Remark 2.2 .

Our goal is to formulate the initial-boundary value problem $(1),(2),(9),(10)$ in a way that its numerical approximation be as simple as possible to implement. We look for $\mathbf{u} \in V, p \in M$ and $\lambda_{1}, \ldots, \lambda_{n} \in \mathbb{R}$ such that, for all $\mathbf{v} \in V$ and $q \in M$,

$$
\left\{\begin{array}{l}
\left(\partial_{t} \mathbf{u}+\mathbf{u} \cdot \nabla \mathbf{u}, \mathbf{v}\right)+\nu(\nabla \mathbf{u}, \nabla \mathbf{v})+\sum_{i=1}^{n} \lambda_{i}<\phi_{i}, \mathbf{v}>-(p, \operatorname{div} \mathbf{v})=<\mathbf{f}, \mathbf{v}> \\
(q, \operatorname{div} \mathbf{u})=0, \\
<\phi_{i}, \mathbf{u}>=Q_{i}, \quad i=1, . ., n,
\end{array}\right.
$$

for all $t>0$, with $\mathbf{u}=\mathbf{u}_{0}$ for $t=0$.

Note that now the test functions $\mathbf{v}$ are taken in $V$, a space which is more straightforward to discretise than $V^{\star}$.

\section{Proposition 1}

Any smooth solution of (11) satisfies the additional boundary conditions

$$
\left.\left(p-\nu \frac{\partial u_{n}}{\partial \mathbf{n}}\right)\right|_{S_{i}}=\lambda_{i}, \quad \text { and }\left.\quad \frac{\partial \mathbf{u}_{\tau}}{\partial \mathbf{n}}\right|_{S_{i}}=0, \quad i=1, \cdots n .
$$

In particular, this yields that both $\frac{\partial \mathbf{u}_{\tau}}{\partial \mathbf{n}}$ and $p-\nu \frac{\partial u_{n}}{\partial \mathbf{n}}$ are indeed constant over $S_{i}$ for $i=1, \cdots, n$. Furthermore, (u, $p$ ) satisfies (1), (2), (9), (10).

\section{Proof.}

Conditions (2) and (9) are obviously satisfied. Integrating by parts the first equation of (11) yields for any $\mathbf{v} \in V$

$$
\begin{aligned}
<\partial_{t} \mathbf{u}+\mathbf{u} \cdot \nabla \mathbf{u}-\nu \Delta \mathbf{u}+ & \nabla p, \mathbf{v}>+\int_{S_{0}}\left(\nu \frac{\partial \mathbf{u}}{\partial \mathbf{n}}-p \mathbf{n}\right) \cdot \mathbf{v} d s \\
& +\sum_{i=1}^{n} \int_{S_{i}}\left(\nu \frac{\partial \mathbf{u}}{\partial \mathbf{n}}-p \mathbf{n}+\lambda_{i} \mathbf{n}\right) \cdot \mathbf{v} d s=\langle\mathbf{f}, \mathbf{v}\rangle
\end{aligned}
$$

Now taking $\mathbf{v} \in \mathcal{D}(\Omega)$, we recover the momentum equation (1) in the sense of $\mathcal{D}^{\prime}(\Omega)$. Consequently, from (13), it follows that

$$
\int_{S_{0}}\left(\nu \frac{\partial \mathbf{u}}{\partial \mathbf{n}}-p \mathbf{n}\right) \cdot \mathbf{v} d s+\sum_{i=1}^{n} \int_{S_{i}}\left(\nu \frac{\partial \mathbf{u}}{\partial \mathbf{n}}-p \mathbf{n}+\lambda_{i} \mathbf{n}\right) \cdot \mathbf{v} d s=0 .
$$

for all $\mathbf{v} \in V$. Using now the splitting of the trace of $u$ on $\partial \Omega$ in its normal and tangential component $\left.\mathbf{u}\right|_{\partial \Omega}=u_{n} \mathbf{n}+\mathbf{u}_{\tau}$, we deduce relations (10) and (12).

$\diamond$

Remark 2.1 Among all possible solutions of (1), (2), (9), (10), problem (11) selects the one that satisfies the additional boundary condition (12).

Remark 2.2 In (11), we could have imposed a flux $Q_{0}$ on $S_{0}$, instead of the homogeneous Neumann condition (10). The corresponding problem would have been: find $\mathbf{u} \in V, \tilde{p} \in M$ and $\tilde{\lambda}_{0}, \tilde{\lambda}_{1}, \ldots, \tilde{\lambda}_{n} \in \mathbb{R}$ such that, for all $\mathbf{v} \in V$ and $q \in M$,

$$
\left\{\begin{array}{l}
\left(\partial_{t} \mathbf{u}+\mathbf{u} \cdot \nabla \mathbf{u}, \mathbf{v}\right)+\nu(\nabla \mathbf{u}, \nabla \mathbf{v})+\sum_{i=0}^{n} \tilde{\lambda}_{i}<\phi_{i}, \mathbf{v}>-(p, \operatorname{div} \mathbf{v})=<\mathbf{f}, \mathbf{v}> \\
(q, \operatorname{div} \mathbf{u})=0, \\
<\phi_{i}, \mathbf{u}>=Q_{i}, \quad i=0, . ., n,
\end{array}\right.
$$


for all $t>0$, with $\mathbf{u}=\mathbf{u}_{0}$ for $t=0$.

Due to the incompressibility of the fluid, the value of $Q_{0}$ must be equal to $-\sum_{i=1}^{n} Q_{i}$ (otherwise the problem has no solution). If $\left(\mathbf{u}, \tilde{p}, \tilde{\lambda}_{0}, \tilde{\lambda}_{1}, \ldots, \tilde{\lambda}_{n}\right)$ is a solution of $(14)$, then, for any constant $C \in \mathbb{R},\left(\mathbf{u}, \tilde{p}+C, \tilde{\lambda}_{0}+C, \tilde{\lambda}_{1}+C, \ldots, \tilde{\lambda}_{n}+C\right)$ is also a solution. The solution of (14) is thus defined up to an additive constant. Now, we set this constant $C$ equal to $-\tilde{\lambda}_{0}$, and we denote $\tilde{p}+C$ and $\tilde{\lambda}_{i}+C$ by $p$ and $\lambda_{i}$. Then, (u, $\left.p, \lambda_{1}, \ldots, \lambda_{n}\right)$ is the solution of (11) and, according to the "implicit" boundary condition (12), $\lambda_{0}=0$ yields nothing but the Neumann boundary condition (10). In other words, problem (11) (with Neumann condition on $S_{0}$ ) and (14) (with flux condition on $S_{0}$ ) are equivalent as soon as the "free" constant of problem (14) is well chosen.

Remark 2.3 From a theoretical viewpoint, our approach is very close to the do-nothing formulation recalled in the previous section. Comparing (8) and (12), we may note that the Lagrange multipliers corresponding to the constraints on the flux are in fact equal to the "a priori unknown" constants of the do-nothing formulation (8). Yet, our approach uses a standard functional space $V$ which can be more straightforwardly discretised than the space $V^{*}$.

Now, for the sake of simplicity, we restrict ourselves to the analysis of the stationary Stokes problem (which embodies however all relevant difficulties of our Lagrange multiplier approach) : find $\left(\mathbf{u}, p, \lambda_{1}, \ldots, \lambda_{n}\right) \in V \times M \times \mathbb{R}^{n}$, such that, $\forall(\mathbf{v}, q) \in V \times M$

$$
\left\{\begin{aligned}
\nu(\nabla \mathbf{u}, \nabla \mathbf{v})+\sum_{i=1}^{n} \lambda_{i}<\phi_{i}, \mathbf{v}>-(p, \operatorname{div} \mathbf{v}) & =<\mathbf{f}, \mathbf{v}> \\
(q, \operatorname{div} \mathbf{u}) & =0 \\
<\phi_{i}, \mathbf{u}> & =Q_{i}, i=1, . ., n .
\end{aligned}\right.
$$

The extension of the analysis to the complete time-dependent, nonlinear problem (11) can then be carried out by usual techniques (see, e.g. $[15,6,13]$ ).

\section{Proposition 2}

Problem (15) is well-posed.

\section{Proof.}

In order to prove existence, let us denote by $(\tilde{\mathbf{u}}, \tilde{p}) \in V \times M$ the solution of

$$
\left\{\begin{aligned}
\nu(\nabla \tilde{\mathbf{u}}, \nabla \mathbf{v})-(\tilde{p}, \operatorname{div} \mathbf{v}) & =<\mathbf{f}, \mathbf{v}> \\
(q, \operatorname{div} \tilde{\mathbf{u}}) & =0
\end{aligned}\right.
$$

for all $(\mathbf{v}, q) \in V \times M$. This is the weak formulation of the Stokes problem with homogeneous Dirichlet conditions on $\Gamma$ and homogeneous Neumann conditions on $\partial \Omega \backslash \Gamma$.

Moreover, for $i=1, \ldots, n$, let $\left(\mathbf{w}_{i}, \pi_{i}\right) \in V \times M$ be the solution of the problem

$$
\left\{\begin{aligned}
\nu\left(\nabla \mathbf{w}_{i}, \nabla \mathbf{v}\right)-\left(\pi_{i}, \operatorname{div} \mathbf{v}\right) & =-<\phi_{i}, \mathbf{v}> \\
\left(q, \operatorname{div} \mathbf{w}_{i}\right) & =0
\end{aligned}\right.
$$

for all $(\mathbf{v}, q) \in V \times M$.

Both systems (16) and (17) admit a unique solution. Note that the equations satisfied by ( $\tilde{\mathbf{u}}, \tilde{p})$ are the unconstrained counterpart of (15) and that the solution $\left(\mathbf{w}_{i}, \pi_{i}\right)$ of $(17)$ depends only on the geometry and not on the data of the Stokes problem. In some sense, the functions $\mathbf{w}_{i}$ are related to the flux carriers $\mathbf{b}_{i}$ introduced in the do-nothing formulation (7).

We set, then, $\mathbf{u}=\tilde{\mathbf{u}}+\sum_{i=1}^{n} \lambda_{i} \mathbf{w}_{i}$ and $p=\tilde{p}+\sum_{i=1}^{n} \lambda_{i} \pi_{i}$. No matter how the $\lambda_{i}, i=1, \ldots, n$, are chosen, $(\mathbf{u}, p)$ satisfies the first two equations of $(15)$. If we further require $\mathbf{u}$ to satisfy the third equation of (15), we obtain the following equations

$$
<\phi_{i}, \tilde{\mathbf{u}}>+\sum_{j=1}^{n} \lambda_{j}<\phi_{i}, \mathbf{w}_{j}>=Q_{i}, \quad i=1, \ldots, n,
$$


whose compact form reads

$$
B \Lambda=Q-\tilde{Q}
$$

where $Q=\left(Q_{1}, \ldots, Q_{n}\right) \in \mathbb{R}^{n}, \quad \tilde{Q}=\left(<\phi_{1}, \tilde{\mathbf{u}}>, \ldots,<\phi_{n}, \tilde{\mathbf{u}}>\right) \in \mathbb{R}^{n}, \quad \Lambda=\left(\lambda_{1}, \ldots, \lambda_{n}\right) \in \mathbb{R}^{n}$ and $B \in \mathbb{R}^{n \times n}, B_{i j}=<\phi_{i}, \mathbf{w}_{j}>$. The matrix $B$ is non singular (as it will be proven in Lemma $2.1)$, thus, once the $\left\{\lambda_{i}\right\}$ are computed through relation $(18),\left(\mathbf{u}, p, \lambda_{1}, \ldots, \lambda_{n}\right)$ will provide a solution of (15).

To prove uniqueness, let $\left(\mathbf{u}_{1}, p_{1}, \lambda_{1}^{(1)}, \ldots, \lambda_{n}^{(1)}\right)$ and $\left(\mathbf{u}_{2}, p_{2}, \lambda_{1}^{(2)}, \ldots, \lambda_{n}^{(2)}\right)$ be two solutions of $(15)$. Then

$$
\left\{\begin{array}{l}
\nu\left(\nabla\left(\mathbf{u}_{1}-\mathbf{u}_{2}\right), \nabla \mathbf{v}\right)+\sum_{i=1}^{n}\left(\lambda_{i}^{(1)}-\lambda_{i}^{(2)}\right)<\phi_{i}, \mathbf{v}>-\left(p_{1}-p_{2}, \operatorname{div} \mathbf{v}\right)=0 \\
\left(q, \operatorname{div}\left(\mathbf{u}_{1}-\mathbf{u}_{2}\right)\right)=0, \\
<\phi_{i}, \mathbf{u}_{1}-\mathbf{u}_{2}>=0, \quad i=1, . ., n .
\end{array}\right.
$$

for all $\mathbf{v} \in V$ and $q \in M$.

Taking $\mathbf{v}=\mathbf{u}_{1}-\mathbf{u}_{2}$ in (19) we obtain $\nu\left\|\nabla\left(\mathbf{u}_{1}-\mathbf{u}_{2}\right)\right\|_{L^{2}(\Omega)}=0$, whence $\mathbf{u}_{1}=\mathbf{u}_{2}$ a.e. in $\Omega$. Consequently,

$$
\sum_{i=1}^{n}\left(\lambda_{i}^{(1)}-\lambda_{i}^{(2)}\right)<\phi_{i}, \mathbf{v}>-\left(p_{1}-p_{2}, \operatorname{div} \mathbf{v}\right)=0, \quad \forall \mathbf{v} \in V
$$

For all $i=1, \ldots, n$ we can construct $\mathbf{w}_{i} \in V$ which satisfy

$$
\operatorname{div} \mathbf{w}_{i}=0,\left.\quad \mathbf{w}_{i}\right|_{S_{j}}=0, j=1, \ldots, n, j \neq i \quad \text { and } \quad\left\langle\phi_{i}, \mathbf{w}_{i}\right\rangle=1 .
$$

Note that $\left\langle\phi_{0}, \mathbf{w}_{i}\right\rangle=-1$ for all $i=1, \ldots, n$.

Taking in (20) $\mathbf{v}=\mathbf{w}_{i}$ we obtain

$$
\lambda_{i}^{(1)}=\lambda_{i}^{(2)}, \quad \forall i=1, \ldots n .
$$

Finally, choosing $\mathbf{z} \in V$ such that

$$
\operatorname{div} \mathbf{z}=p_{1}-p_{2},\left.\quad \mathbf{z}\right|_{S_{i}}=0 \forall i=1, \ldots, n,
$$

(such a function exists, see e.g. $[6,9]$ ) and taking $\mathbf{v}=\mathbf{z}$ in $(20)$, we obtain $\left\|p_{1}-p_{2}\right\|_{L^{2}(\Omega)}=0$. Henceforth $p_{1}=p_{2}$ a.e. in $\Omega$. $\diamond$

Lemma 2.1 The matrix B introduced in (18) is non singular.

Proof. Given an arbitrary vector $\boldsymbol{\alpha} \in \mathbb{R}^{n}$, for any $i=1, \ldots, n$ we can multiply each problem (17) by $\alpha_{i}$ and sum from $i=1$ to $n$. Owing to the linearity of (17) we have

$$
\left\{\begin{aligned}
\nu\left(\sum_{i=1}^{n} \alpha_{i} \nabla \mathbf{w}_{i}, \nabla \mathbf{v}\right)-\left(\sum_{i=1}^{n} \alpha_{i} \pi_{i}, \operatorname{div} \mathbf{v}\right)+\sum_{i=1}^{n} \alpha_{i}<\phi_{i}, \mathbf{v}> & =0 \\
\left(q, \operatorname{div} \sum_{i=1}^{n} \alpha_{i} \mathbf{w}_{i}\right) & =0
\end{aligned}\right.
$$

Now taking in $\mathbf{v}=\sum_{i=1}^{n} \alpha_{i} \mathbf{w}_{i}$, we obtain

$$
\nu\left\|\nabla\left(\sum_{i=1}^{n} \alpha_{i} \mathbf{w}_{i}\right)\right\|_{L^{2}(\Omega)}^{2}+\sum_{i=1}^{n} \alpha_{i}<\phi_{i}, \sum_{j=1}^{n} \alpha_{j} \mathbf{w}_{j}>=0,
$$


which implies, for all $\boldsymbol{\alpha} \in \mathbb{R}^{n}, \boldsymbol{\alpha} \neq \mathbf{0}$,

$$
\boldsymbol{\alpha}^{T} B \boldsymbol{\alpha}=-\nu\left\|\nabla\left(\sum_{i=1}^{n} \alpha_{i} \mathbf{w}_{i}\right)\right\|_{L^{2}(\Omega)}^{2} \leq-\frac{\nu}{1+C_{p}}\left\|\sum_{i=1}^{n} \alpha_{i} \mathbf{w}_{i}\right\|_{H^{1}(\Omega)}^{2}<0 .
$$

In the last relation we have used the Poincaré inequality

$$
\int_{\Omega} \mathbf{v}^{2} \leq C_{p} \int_{\Omega}|\nabla \mathbf{v}|^{2}, \forall \mathbf{v} \in V
$$

From (22) we infer that $B$ is negative definite and then non-singular. $\diamond$

Remark 2.4 Let us point out a difficulty that may be encountered if one wants to impose on the sections $S_{i}$ a mean value $P \in \mathbb{R}$ for the pressure (or, for the normal stresses) by following a route similar to that presented for the mass flux (the case where one wants to impose a pointwise value for the pressure on the boundary for the Stokes problem has been analysed in [1]). For the sake of simplicity, we restrict ourselves to the Stokes problem. A possible formulation is: find $(\mathbf{u}, p) \in V \times M$ and $\lambda_{0}, \ldots, \lambda_{n} \in \mathbb{R}$ such that, $\forall(\mathbf{v}, q) \in V \times M$,

$$
\left\{\begin{aligned}
\nu(\nabla \mathbf{u}, \nabla \mathbf{v})+(\nabla p, \mathbf{v}) & =<\mathbf{f}, \mathbf{v}> \\
(\nabla q, \mathbf{u})-\sum_{i=0}^{n} \lambda_{i} \int_{S_{i}} q d s & =0 \\
\int_{S_{i}} p d s & =P_{i} \operatorname{meas}\left(S_{i}\right), \quad i=0, \ldots, n .
\end{aligned}\right.
$$

This formulation is, in some sense, the dual of the flux formulation as it imposes constraints on the dual problem (the pressure equation), whereas the flux boundary conditions yields a constraint on the primal problem (the velocity equations). Therefore, it can be regarded as the natural counterpart of our formulation for the flux problem. Unfortunately, it may be recognised that from (23) it follows that $\left.\mathbf{u} \cdot \mathbf{n}\right|_{S_{i}}=\lambda_{i}$ on each $S_{i}$, whereas $\left.\mathbf{u} \cdot \mathbf{n}\right|_{S_{i}}$ cannot be a constant different from 0 (since we assume no-slip boundary conditions on $\Gamma$ ). This formulation is therefore not well-suited for the problem in which we are interested. Nevertheless, it may be adopted in those cases where a slip boundary condition is imposed on the wall. In this case system (23) will effectively impose a mean value for $p$, thus differing from the do-nothing approach (5) which is instead equivalent to impose the much stronger condition (6).

\section{The numerical solution of the Lagrange multipliers prob- lem}

In order to discretise formulation (15), we introduce a Galerkin approximation based on the finite dimensional spaces $V_{h} \subset V$ and $M_{h} \subset M$, which we assume to satisfy the well-known LBB condition

$$
\forall q_{h} \in M_{h} \quad \exists \mathbf{v}_{h} \in V_{h}, \mathbf{v}_{h} \neq 0: \quad\left(q_{h}, \operatorname{div} \mathbf{v}_{h}\right) \geq \beta_{h}\left\|q_{h}\right\|_{L^{2}}\left\|\mathbf{v}_{h}\right\|_{H^{1}} .
$$

Let $\left(\mathbf{u}_{h}, p_{h}, \lambda_{1 h}, \ldots, \lambda_{n h}\right)$ be the solution of the discrete problem. We denote by $\left(u_{i}\right)_{i=1 . . d N}$ (resp. $\left.\left(p_{i}\right)_{i=1 . . M}\right)$ the components of $\mathbf{u}_{h}$ (resp. $p_{h}$ ) with respect to a basis $\left\{\mathbf{v}_{i}\right\}$ of $V_{h}$ (resp. $\left\{q_{i}\right\}$ of $\left.M_{h}\right)$. Finally, we introduce the vectors $U=\left(u_{1}, . ., u_{d N}\right) \in \mathbb{R}^{d N}, P=\left(p_{1}, . ., p_{M}\right) \in \mathbb{R}^{M}$ and $\Lambda=\left(\lambda_{1 h}, \ldots, \lambda_{n h}\right) \in \mathbb{R}^{n}$.

Then the discrete counterpart of (15) gives rise to the following algebraic system of equations

$$
\left\{\begin{aligned}
A U+D^{T} P+\Phi^{T} \Lambda & =F \\
D U & =0 \\
\Phi U & =Q
\end{aligned}\right.
$$


where $A \in \mathbb{R}^{d N \times d N}$ is the stiffness matrix, $D \in \mathbb{R}^{M \times d N}$ is the matrix associated to the divergence operator and $\Phi$ is the $n \times d N$ matrix whose lines are given by the vectors $\phi_{i}=\left(\int_{S_{i}} \mathbf{v}_{1}\right.$. $\left.\mathbf{n} d s, \ldots, \int_{S_{i}} \mathbf{v}_{d N} \cdot \mathbf{n} d s\right), i=1, \ldots, n$.

\section{Proposition 3}

System (25) admits a unique solution.

Proof. The proof of the existence of the discrete solution $\left(\mathbf{u}_{h}, p_{h}, \lambda_{i h}, i=1, \cdots, n\right)$ as well as that of the uniqueness of $\mathbf{u}_{h}$ and of the $\lambda_{i h}$ follows the same lines of Proposition 2, by substituting $V$ and $M$ by their discrete counterpart. The uniqueness of $p_{h}$ is assured by condition (24). $\diamond$

If we discretise in time the Navier-Stokes system (11) by, for instance, a semi-implicit Euler scheme, and then in space by the finite element method, we will produce an algebraic system analogous to (25), where the matrix $A$ is now given by

$$
A=\frac{1}{\delta t} M+B+K
$$

$\delta t$ being the time-step, $M$ and $B$ the mass and advection matrices and $K$ the stiffness matrix. Here and in the following it is assumed that the matrix $A$ is positive definite, which is always the case if $\delta t$ is chosen appropriately.

\subsection{Solution algorithms}

Here, we present and analyse four possible algorithms which may be adopted for the solution of system (25) and which are computationally more efficient than solving simultaneously for $U, P$ and $\Lambda$.

\section{Solution of additional Stokes problems}

If one wishes to introduce the proposed approach on a Navier-Stokes solver with as little modifications as possible to an existing code for Navier-Stokes equations with "classical" boundary conditions, a possibility is to follow the constructive proof of Proposition 2. As we have seen, the solution of the constrained problem can be obtained by combining the solutions of $n+1$ unconstrained Stokes problems, given by (16) and (17). In particular, for a fixed geometry, the $n$ solutions $\mathbf{w}_{i}, i=1, \ldots, n$ of problem (17) can be computed only once, so that the additional computational cost at each time step is just that of the solution of (16). The drawback is that the memory requirement to store the $\mathbf{w}_{i}$ may become prohibitive particularly in a $3 \mathrm{D}$ computation and with a large number of Lagrange multipliers.

\section{Schur complement + Iterative Solver}

An alternative algorithm is based on using an iterative solution of a Schur complement system. We rewrite (25) in the form

$$
\left[\begin{array}{cc}
S & \tilde{\Phi}^{T} \\
\tilde{\Phi} & 0
\end{array}\right],\left[\begin{array}{l}
X \\
\Lambda
\end{array}\right]=\left[\begin{array}{l}
G \\
Q
\end{array}\right]
$$

where $\tilde{\Phi}=[\Phi, 0] \in \mathbb{R}^{n \times(d N+M)}, X=[U, P]^{T}, G=[F, 0]^{T}$. The matrix

$$
S=\left[\begin{array}{cc}
A & D^{T} \\
D & 0
\end{array}\right]
$$

has a standard Stokes form and, since the two discrete spaces $V_{h}$ and $M_{h}$ satisfy the LBB condition (24), $S$ is non singular (see, e.g. $[13,2]$ ). We can then eliminate the unknown $X$ from (26), obtaining

$$
\tilde{\Phi} S^{-1} \tilde{\Phi}^{T} \Lambda=\tilde{\Phi} S^{-1} G-Q
$$


which can be solved by an appropriate iterative method. Any matrix-vector multiplication will imply the solution of a Stokes problem with homogeneous Neumann conditions on the sections $S_{i}$. If $A$ is symmetric, then $R=\tilde{\Phi} S^{-1} \tilde{\Phi}^{T}$ is symmetric and positive definite (see Proposition 4 below). Consequently, the Conjugate Gradient (CG) algorithm may be used, which will converge to the exact solution in $n$ iterations, being $n$ the number of Lagrange multipliers (which coincides with the number of boundary sections $S_{i}$ minus one). For instance, in the case of just one Lagrange multiplier, one iteration of the CG algorithm suffices to obtain the solution (note that in this case the linear system (27) reduces to just one scalar equation).

Remark 3.1 The computational cost of the procedure depends on the number of matrix-vector multiplications required for every iteration of the chosen iterative solver and on the number of iterations necessary for convergence. For each matrix-vector multiplication we need to solve a Stokes problem. In the case of the CG algorithm, it is known that it converges to the exact solution in $n$ steps. In addition, two extra Stokes problems have to be solved to obtain the initial residual (required to start up the procedure) and the final solution $X$. Therefore, if CG is adopted the computational cost would be equal to the solution of $n+2$ Stokes problems (at each time step), which is higher than that of Procedure 1. On the other hand, there is no need to store intermediate solutions.

\section{Proposition 4}

The matrix $R=\tilde{\Phi} S^{-1} \tilde{\Phi}^{T}$ is positive semidefinite; moreover, if $A$ is symmetric then $R$ is symmetric and positive definite.

Proof. System (26) (which is equivalent to system (25)) admits a unique solution, as shown in Proposition 3. Then, we have necessarily that $\operatorname{ker}\left(\tilde{\Phi}^{T}\right)=\{0\}$.

The matrix

$$
S^{*}=\left[\begin{array}{cc}
A & D^{T} \\
-D & 0
\end{array}\right]
$$

is positive semidefinite (see e.g. [13]), thus $S^{*-1}$ and $R^{*}=\tilde{\Phi} S^{*-1} \tilde{\Phi}^{T}$ are also positive semidefinite. On the other hand, $S=P S^{*}$, where

$$
P=\left[\begin{array}{cc}
I & 0 \\
0 & -I
\end{array}\right]
$$

is such that $P^{-1}=P$. Then,

$$
R=\tilde{\Phi} S^{-1} \tilde{\Phi}^{T}=\tilde{\Phi}\left(P S^{*}\right)^{-1} \tilde{\Phi}^{T}=\tilde{\Phi} S^{*-1} P \tilde{\Phi}^{T}=\tilde{\Phi} S^{*-1} \tilde{\Phi}^{T}=R^{*} .
$$

We conclude that also $R$ is positive semidefinite. Moreover, if $A$ is symmetric, $R$ turns out to be symmetric, positive semidefinite and non-singular, so it is also positive definite. $\diamond$

In the case where there is just one Lagrange multiplier (which occurs whenever we have just one input section and one output section), the CG algorithm reads as follows :

given $\lambda_{0} \in \mathbb{R}$,

$$
\begin{aligned}
& S X_{1}=G-\tilde{\Phi}^{T} \lambda_{0} \\
& r_{0}=\tilde{\Phi} X_{1}-Q \\
& S X_{2}=\tilde{\Phi}^{T} r_{0} \\
& \lambda=\lambda_{0}+\frac{r_{0}^{2}}{r_{0} \tilde{\Phi} X_{2}} r_{0}=\lambda_{0}+\frac{r_{0}^{2}}{\tilde{\Phi} X_{2}} \\
& S X=G-\tilde{\Phi}^{T} \lambda
\end{aligned}
$$

Since in this case the CG method converges in one iteration, $\lambda$ and $X$ are the solutions of (26). This algorithm requires the solution of 3 Stokes problems at steps $(i),(i i i)$ and $(v)$. 
Remark 3.2 By a closer inspection, it can be noted that by taking $\lambda_{0}=0$, the CG algorithm just presented effectively reduces to procedure 1 .

\section{Reordering + fractional step I}

We recall that any Stokes system of the form

$$
\left[\begin{array}{cc}
A & D^{T} \\
D & 0
\end{array}\right]\left[\begin{array}{l}
U \\
P
\end{array}\right]=\left[\begin{array}{l}
F \\
0
\end{array}\right]
$$

can be solved exactly by the following three step algorithm :

(i) $A U_{0}=F$,

(ii) $D A^{-1} D^{T} P=D U_{0}$,

(iii) $U=U_{0}-A^{-1} D^{T} P$.

Let $H^{(1)}$ and $H^{(2)}$ denote two suitable approximations of $A^{-1}$. We can write an approximate factorization scheme as

(i) $A U_{0}=F$,

(ii) $D H^{(1)} D^{T} P=D U_{0}$,

(iii) $U=U_{0}-H^{(2)} D^{T} P$.

In this way we can recover many projection or quasi-compressibility methods for the Navier-Stokes equations (see $[10,14])$. In particular, we will focus on the Yosida projection scheme ([11]) which consists in adopting $H^{(1)}=\delta t M^{-1}$ (while no approximation is made in step (iii), i.e. $H^{(2)}=A^{-1}$ ), and on the algebraic version of the Chorin-Temam scheme in which we have $H^{(1)}=H^{(2)}=\delta t M^{-1}$ [14].

System (25) can be reordered in a Stokes-like form as

$$
\left[\begin{array}{cc}
\tilde{A} & \tilde{D}^{T} \\
\tilde{D} & 0
\end{array}\right]\left[\begin{array}{c}
\tilde{U} \\
P
\end{array}\right]=\left[\begin{array}{c}
\tilde{F} \\
0
\end{array}\right]
$$

where

$$
\begin{aligned}
\tilde{A} & =\left[\begin{array}{cc}
A & \Phi^{T} \\
\Phi & 0
\end{array}\right] \in \mathbb{R}^{(d N+n) \times(d N+n)}, & \tilde{D} & =\left[\begin{array}{ll}
D & 0
\end{array}\right] \in \mathbb{R}^{M \times(d N+n)}, \\
\tilde{U} & =\left[\begin{array}{l}
U \\
\Lambda
\end{array}\right] \in \mathbb{R}^{d N+n}, & \tilde{F} & =\left[\begin{array}{l}
F \\
Q
\end{array}\right] \in \mathbb{R}^{d N+n}
\end{aligned}
$$

We can then write an approximate factorisation scheme as follows.

(i) $\tilde{A} \tilde{U}_{0}=\tilde{F}$

Since $A$ is positive definite and $\operatorname{ker}\left(\Phi^{T}\right)=\emptyset, \tilde{A}$ is non singular too and this system admits a unique solution. In particular, we have $\Phi U_{0}=Q$.

(ii) $\tilde{D} \tilde{H}^{(1)} \tilde{D}^{T} P=\tilde{D} \tilde{U}_{0}$.

(iii) $\tilde{U}=\tilde{U}_{0}-\tilde{H}^{(2)} \tilde{D}^{T} P$

where now $\tilde{H}^{(1)}$ and $\tilde{H}^{(2)}$ are possible approximations of $\tilde{A}^{-1}$. 
We are now in the same setting of factorization schemes for the Stokes problem. We will then use the term Yosida scheme when $\tilde{A}^{-1}$ is approximated only in step (ii), while a scheme where $\tilde{H}^{(1)}=\tilde{H}^{(2)} \neq \tilde{A}^{-1}$ will be called a Chorin-Temam scheme.

We now detail a possible way to approximate $\tilde{A}^{-1}$. Firstly, we note that if we write $\tilde{H}^{(1)}$ in the following block form

$$
\tilde{H}^{(1)}=\left[\begin{array}{ll}
H_{11}^{(1)} & H_{12}^{(1)} \\
H_{21}^{(1)} & H_{22}^{(1)}
\end{array}\right],
$$

step $(i i)$ of the algorithm is equivalent to

$$
D H_{11}^{(1)} D^{T} P=D U_{0},
$$

where just the first diagonal block of $\tilde{H}^{(1)}$ is actually involved in the computation. Therefore, we only need to look for and approximation $H_{11}^{(1)}$ of the corresponding term $C_{11}$ of the following block decomposition of $\tilde{A}^{-1}$

$$
\tilde{A}^{-1}=\left[\begin{array}{ll}
C_{11} & C_{12} \\
C_{21} & C_{22}
\end{array}\right]
$$

The term $C_{11}$ is equal to

$$
C_{11}=A^{-1}\left(I-\Phi^{T} V^{-1} \Phi A^{-1}\right), \quad \text { where } V=\Phi A^{-1} \Phi^{T} .
$$

A natural approximation of $C_{11}$ is then

$$
H_{11}^{(1)}=\delta t M^{-1}\left(I-\delta t \Phi^{T} V_{a p p r}^{-1} \Phi M^{-1}\right), \quad V_{a p p r}=\delta t \Phi M^{-1} \Phi^{T},
$$

and in particular we have $H_{11}^{(1)}=C_{11}+O\left(\delta t^{2}\right)$. The matrix $V_{a p p r}$ is an $n \times n$ matrix. In general, the number $n$ of Lagrange multipliers is very small so that $V_{a p p r}$ can be easily inverted. Furthermore, if the lumped form of the mass matrix is used, $V_{a p p r}$ is diagonal. In the case of just one Lagrange multiplier, $V_{\text {appr }}$ reduces to a scalar.

The Yosida scheme becomes then

(i) $\tilde{A} \tilde{U}_{0}=\tilde{F}$,

(ii) $D H_{11}^{(1)} D^{T} P=D U_{0}$ and

(iii) $\tilde{A} \tilde{U}=\tilde{A} \tilde{U}_{0}-\tilde{D}^{T} P$

We observe that, in this case, we recover exactly the constraints on the fluxes. Indeed, step (iii) implies in particular $\Phi U=\Phi U_{0}=Q$.

In the Chorin-Temam case, we note that step (iii) is equivalent to

$$
\begin{aligned}
& U=U_{0}-H_{11}^{(1)} D^{T} P, \\
& \Lambda=\Lambda_{0}-H_{21}^{(1)} D^{T} P .
\end{aligned}
$$

Since we are only interested in the velocity field, we can neglect equations (31) and we need only to compute the block $H_{11}^{(1)}$ as in (29). The algebraic Chorin-Temam scheme becomes then:

(i) $\tilde{A} \tilde{U}_{0}=\tilde{F}$,

(ii) $D H_{11}^{(1)} D^{T} P=D U_{0}$ and

(iii) $U=U_{0}-H_{11}^{(1)} D^{T} P$ 
We observe that, also in this case, the constraints on the fluxes are recovered exactly. Indeed, by multiplying step (iii) by $\Phi$ we have

$$
\Phi U=\Phi U_{0}-\Phi H_{11}^{(1)} D^{T} P \quad=\Phi U_{0}-\left(\delta t \Phi M^{-1}-\delta t V_{a p p r} V_{a p p r}^{-1} \Phi M^{-1}\right) D^{T} P=\Phi U_{0}=Q
$$

\section{Reordering + fractional step II}

System (25) can also be reordered in a different manner as

$$
\left[\begin{array}{cc}
A & \tilde{D}^{T} \\
\tilde{D} & 0
\end{array}\right]\left[\begin{array}{l}
U \\
\tilde{P}
\end{array}\right]=\left[\begin{array}{l}
F \\
\tilde{Q}
\end{array}\right]
$$

where

$$
\tilde{D}=\left[\begin{array}{l}
D \\
\Phi
\end{array}\right] \in \mathbb{R}^{(M+n) \times d N}, \quad \tilde{P}=\left[\begin{array}{l}
P \\
\Lambda
\end{array}\right] \in \mathbb{R}^{M+n}, \quad \tilde{Q}=\left[\begin{array}{l}
0 \\
Q
\end{array}\right] \in \mathbb{R}^{M+n}
$$

The three steps algorithm reads then

(i) $A U_{0}=F$

which is unperturbed with respect to the Stokes system without constraints.

(ii) $\tilde{D} H^{(1)} \tilde{D}^{T} \tilde{P}=\tilde{D} U_{0}-\tilde{Q}$

(iii) $U=U_{0}-H^{(2)} \tilde{D}^{T} \tilde{P}$.

Again, we consider the approximation $H^{(1)}=\delta t M^{-1}$ and either $H^{(2)}=A^{-1}$ (Yosida) or $H^{(2)}=$ $\delta t M^{-1}$ (algebraic Chorin-Temam).

Remark 3.3 This algorithm can be easily implemented starting from an existing Navier-Stokes solver which uses factorization methods. It suffices to add to the matrix $D$ the few lines of matrix $\Phi$, and apply the chosen factorization method.

Remark 3.4 Step (ii) is equivalent to

$$
\begin{aligned}
& D H^{(1)}\left(D^{T} P+\Phi^{T} \Lambda\right)=D U_{0} \\
& \Phi H^{(1)}\left(D^{T} P+\Phi^{T} \Lambda\right)=\Phi U_{0}-Q .
\end{aligned}
$$

On the other hand, the third step gives

$$
U=U_{0}-H^{(2)}\left(D^{T} P+\Phi^{T} \Lambda\right)
$$

from which we can infer

$$
\begin{aligned}
\Phi U=\Phi U_{0}-\Phi H^{(2)}\left(D^{T} P+\right. & \left.\Phi^{T} \Lambda\right)= \\
& \Phi U_{0}-\Phi H^{(1)}\left(D^{T} P+\Phi^{T} \Lambda\right)+\Phi\left(H^{(1)}-H^{(2)}\right)\left(D^{T} P+\Phi^{T} \Lambda\right) .
\end{aligned}
$$

By exploiting (34), we finally have

$$
\Phi U=Q+\Phi\left(H^{(1)}-H^{(2)}\right)\left(D^{T} P+\Phi^{T} \Lambda\right)
$$

Whenever $H^{(1)}=H^{(2)}$, like in the algebraic Chorin-Temam scheme, we recover the constraint on the fluxes exactly.

On the contrary, in the Yosida scheme, equation (36) becomes

$$
\Phi U=Q+\Phi\left(\delta t M^{-1}-A^{-1}\right)\left(D^{T} P+\Phi^{T} \Lambda\right)=Q+O\left(\delta t^{2}\right) .
$$




\section{Numerical tests and algorithm assessment}

\subsection{Womersley flow}

In order to assess the proposed methodology, we consider a case where the analytical solution of the Navier-Stokes equations is known. More precisely, we consider the Womersley solution, which describes the transient flow in a cylindrical pipe associated to a time-periodic pressure gradient (see e.g. [8]). As such, it may be considered as a transient counterpart of the Poiseuille solution. If the pressure gradient is given by

$$
\nabla p=\frac{d p}{d z}(t) \mathbf{e}_{z}=-\rho a \sin (\omega t) \mathbf{e}_{z},
$$

$z$ being the pipe axial coordinate and $\rho$ the fluid density, the velocity $\mathbf{u}$ reduces only to its axial component, i.e. $\mathbf{u}=u_{z} \mathbf{e}_{z}$, and the analytical expression for $u_{z}$ is

- $2 D$ case (flow between two infinite planes):

$$
u_{z}(r, t)=\sum_{0}^{\infty} \gamma_{2 k+1} \sin \left(\frac{(2 k+1) \pi}{2 r_{0}} r\right)
$$

where

$$
\gamma_{l}=\frac{4 a}{\pi l\left(l^{4} \sigma^{2}+\omega^{2}\right)}\left(l^{2} \sigma \sin (\omega t)+\omega e^{-l^{2} \sigma t}-\omega \cos (\omega t)\right) .
$$

Here $\sigma=\frac{\mu \pi^{2}}{4 \rho r_{0}^{2}}, r$ is the transversal coordinate, $2 r_{0}$ the distance between the two planes and $\mu$ the dynamic fluid viscosity.

- $3 D$ case (flow in a cylindrical pipe):

$$
u_{z}(r, t)=\operatorname{Re}\left\{-\frac{a}{\omega}\left(1-\frac{J_{0}\left(i^{3 / 2} \sqrt{\frac{\rho \omega}{\mu}} r\right)}{J_{0}\left(i^{3 / 2} \sqrt{\frac{\rho \omega}{\mu}} r_{0}\right)}\right) e^{i \omega t}\right\}
$$

where $r$ is the radial coordinate, $r_{0}$ the cylinder radius and $J_{0}$ the Bessel function of first kind and of order zero.

In both the $2 \mathrm{D}$ and $3 \mathrm{D}$ test cases, we have imposed homogeneous Neumann boundary conditions at the inflow, while at the outflow we have prescribed the mass flux associated to the Womersley solution. In Figure 2 we show the axial velocity field for the 2D case at two different times, together with the velocity profile at the inflow. The solution obtained agrees very well with the analytical Womersley solution. Therefore, a single condition on the mass flux at the outflow, imposed through a Lagrange multiplier, is sufficient to recover the Womersley flow.

The same experiment has been carried out in 3D and the result is shown in Figure 3. Here, the computed velocity field at three different times is illustrated, together with the corresponding axial velocity profile on the inflow section. Again, we outline the excellent agreement with the analytical solution.

Finally, we have carried out the same experiment in 2D using the numerical schemes "reordering + fractional step I" and "reordering + fractional step II" proposed in the previous section, both for the Yosida and the algebraic Chorin-Temam approximations, and we have evaluated the errors introduced on the fluxes. As expected, for the first scheme the difference between the flux we wish to impose and the one actually computed is of the order of the machine round-off error, both for the Yosida and the Chorin-Temam approximation.

For the latter scheme, instead, this is true only when adopting the Chorin-Temam approximation. The behaviour of the error on the fluxes for the Yosida approximation is shown in figure 4. The error is decreasing with the time step size, with a convergence rate that appears to be even higher than quadratic. 

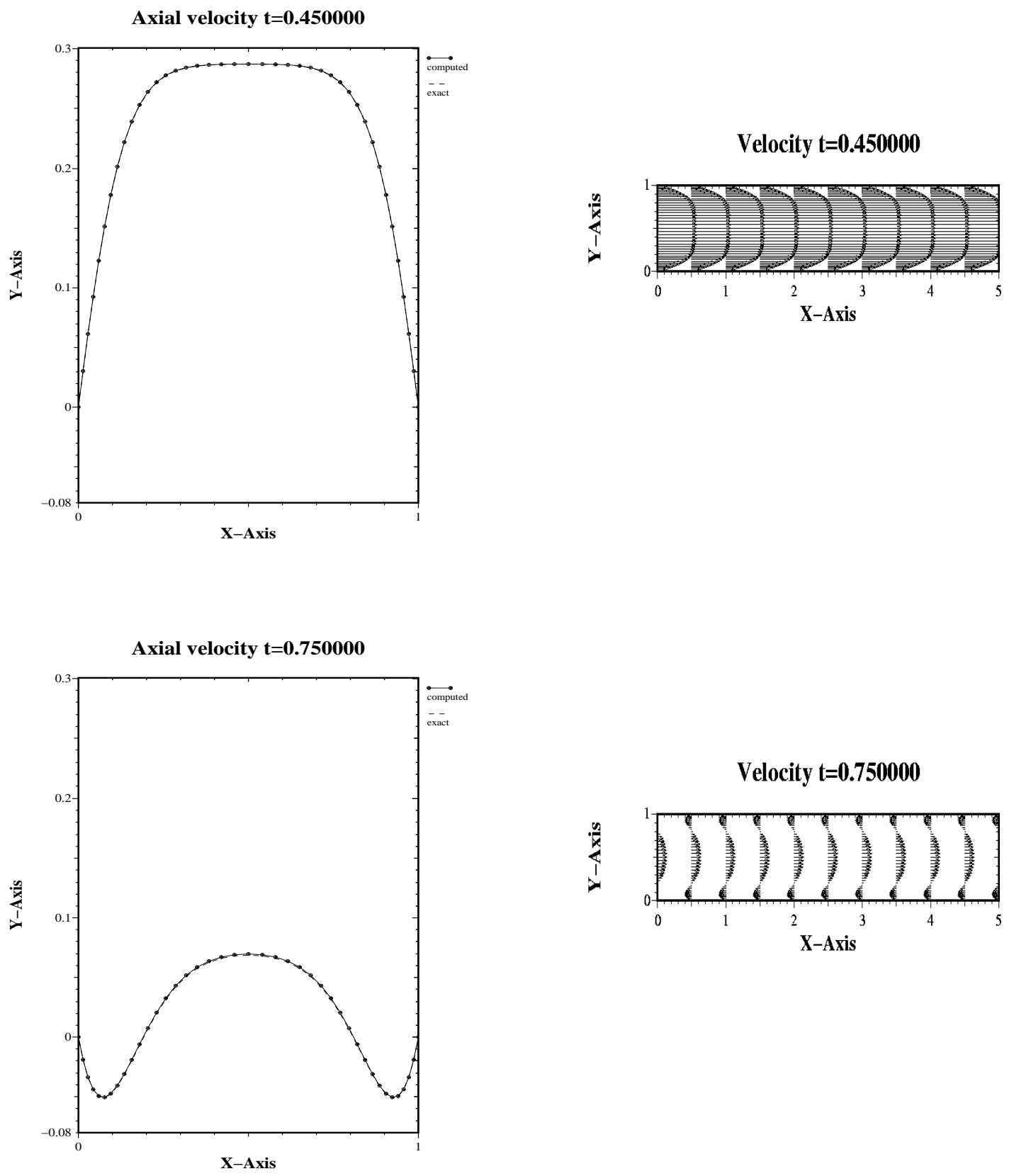

Figure 2: 2D numerical solution obtained imposing the flux of the Womersley solution at the outflow section. 

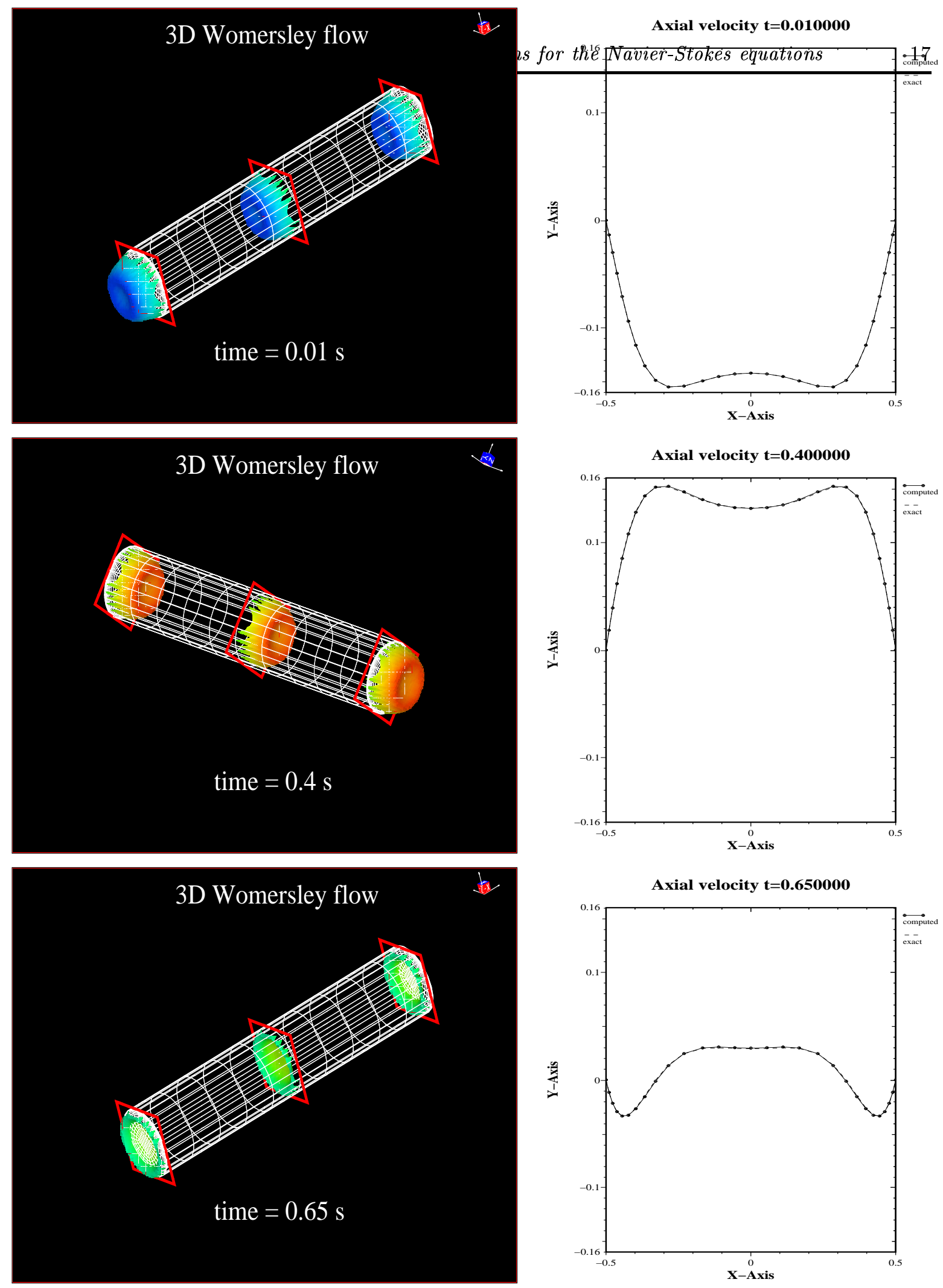

Figure 3: 3D numerical solution obtained imposing the flux of the Womersley solution at the outflow section. 


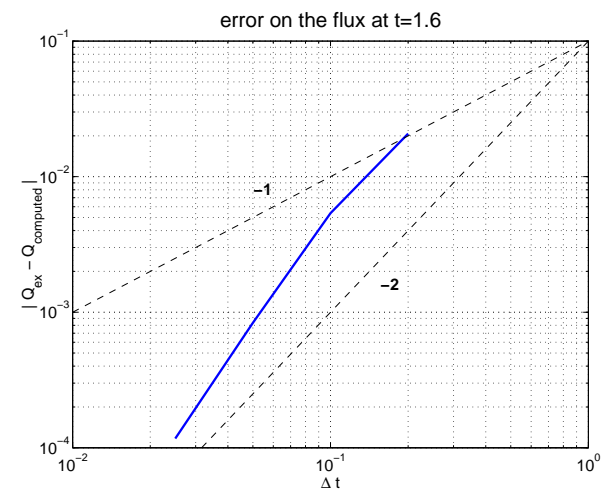

Figure 4: Behaviour of the error on the flux for the scheme "reordering + fractional step II" with the Yosida approximation; the dotted lines are the reference lines for the error decrease rate.

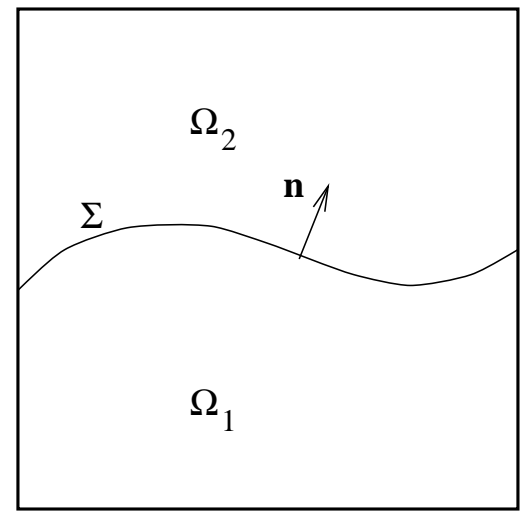

Figure 5: Two fluids separated by a free interface

\subsection{Mass conservation in free interface simulations}

We present here another application where it may be useful to impose the mass flux on a surface. We consider two immiscible and incompressible fluids, with the same viscosity, confined in a closed tank $\Omega$ and separated by a free interface (see Fig. 4.2). We denote by $\Sigma(t)$ the interface at time $t$ and by $\Omega_{i}(t)$ the domain occupied by the fluid $i$ at time $t$, for $i=1,2$. We adopt an Arbitrary Lagrangian Eulerian (ALE) formulation [4] and we denote by $\mathbf{w}$ the domain velocity which satisfies $\mathbf{w} \cdot \mathbf{n}=\mathbf{u} \cdot \mathbf{n}$ on $\Sigma(t)$ and $\mathbf{w} \cdot \boldsymbol{\nu}=0$ on $\partial \Omega$, where $\mathbf{n}$ denotes the normal to $\Sigma(t)$ directed from $\Omega_{1}(t)$ to $\Omega_{2}(t)$ and $\nu$ is the outward normal on $\partial \Omega$. Because of the incompressibility and the immiscibility of the two fluids, the volume of $\Omega_{1}(t)$ (or equivalently $\Omega_{2}(t)$ ) must be preserved. At the continuous level, this property is immediately derived by noting that

$$
\begin{aligned}
\operatorname{meas}\left(\Omega_{1}\left(t_{2}\right)\right)-\operatorname{meas}\left(\Omega_{1}\left(t_{1}\right)\right) & =\int_{t_{1}}^{t_{2}} \int_{\Sigma(t)} \mathbf{w} \cdot \mathbf{n} d \sigma=\int_{t_{1}}^{t_{2}} \int_{\Sigma(t)} \mathbf{u} \cdot \mathbf{n} d \sigma \\
& =\int_{t_{1}}^{t_{2}} \int_{\Omega_{1}(t)} \operatorname{div} \mathbf{u} d x=0
\end{aligned}
$$

since div $\mathbf{u}$ vanishes almost everywhere in $\Omega_{1}(t)$.

At discrete level, the relation $\int_{\Omega_{1}(t)} \operatorname{div} \mathbf{u}_{h} d x=0$ is still verified if the pressure is discretised using discontinuous functions, (as in the Q2/P1 or Q1/P0 finite elements), since we are allowed to choose for the continuity equations a test function equal to 1 in $\Omega_{1}(t)$ and to 0 in $\Omega_{2}(t)$ and consequently (37) is satisfied also after space discretisation. 
If instead the pressure is discretised using continuous functions, as in Taylor-Hood, P1-isoP2 or Q1/Q1 stabilized finite elements [6, 13], there is no guarantee that (37) still holds at the discrete level. Numerical tests indeed confirm that those discretisations fail to conserve meas $\left(\Omega_{1}\right)$. A possible strategy for the solution of this problem is to impose the condition

$$
\int_{\Sigma(t)} \mathbf{u}_{h} \cdot \mathbf{n}_{h} d \sigma=0,
$$

by a Lagrange multiplier, using the techniques presented in the previous section.

Let us show the results obtained on a $2 \mathrm{D}$ test case. In the following, all quantities are given in the International System (IS) units. The two fluids are initially at rest and they are subjected to an oscillating body force $\mathbf{f}=(a g \sin (2 \pi \nu t),-g)$ with $a=0.05, g=10, \nu=0.0625$. The kinematic viscosity of both fluids is taken to be $\nu=0.005$, the density of the upper and the lower fluid is 0.9 and 1 , respectively and at $t=0$ meas $\left(\Omega_{1}\right)=\operatorname{meas}\left(\Omega_{2}\right)=4$. The mesh is allowed to move only along the vertical direction and the typical size of the mesh elements is $h=0.05$. At $t=80$, if we use Q1/P0 or Q2/P1 elements meas $\left(\Omega_{1}\right)$ is still equal to 4 (within machine precision), whereas it drops to approximately 3.4 when we adopt stabilized Q1/Q1 elements (see Figure 6). We noted that the stabilisation is not responsible of this undesired "numerical mass transfer" since we have obtained analogous results with the Q2/Q1 continuous elements. Clearly, this lack of mass conservation decreases as $h$ goes to zero, yet for many practical application a mass loss is not acceptable and the use of an extremely fine mesh is not feasible or economical.

Figure 6 shows that a perfect mass conservation is obtained also with stabilized Q1/Q1 elements if we impose a zero mass flux through $\Sigma$ by the Lagrange multiplier technique. Finally, Figure 7 shows the elevation of a point on the interface, obtained on the same mesh with the Q1/P0 elements and the stabilized Q1/Q1 elements with flux constraint. The difference is barely visible. The use of a Lagrange multiplier technique thus allows to adopt continuous pressure elements for this type of problems.

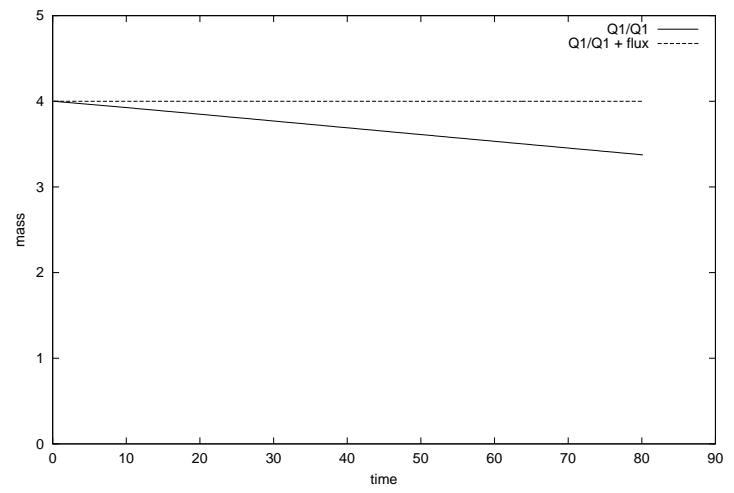

Figure 6: Mass conservation fails using Q1/Q1 elements, while it holds if we add a flux constraint.

\subsection{Multiscale domain decomposition}

An application in which is necessary to impose defective boundary conditions to a Navier-Stokes problem arises in the hemodynamics context when the cardiovascular system is simulated by a multiscale model. A multiscale technique couples detailed models, based on the solution of 2D or 3D fluid-structure interaction problems, with reduced models based on one-dimensional approximations or on systems of ordinary differential equations [5]. The simpler models normally provide the evolution of mean pressure and mean velocity in various regions of the cardiovascular system. The boundary data for the detailed model, which is based on the solution of the NavierStokes equations coupled with the vessel wall dynamics, must be obtained from these averaged quantities. This is a typical case of defective boundary conditions. 


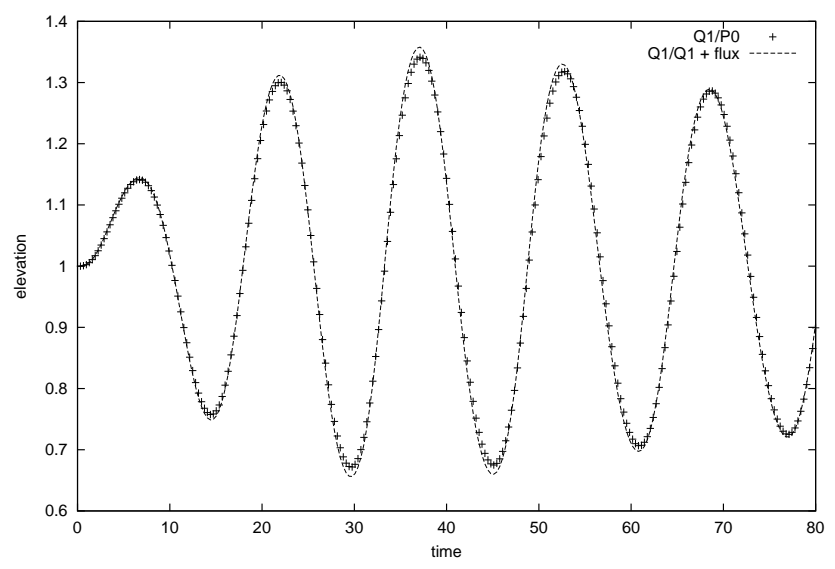

Figure 7: The time history of the elevation of a point of the interface. The result obtained with Q1/P0 elements are almost the same of that given by Q1/Q1 elements with flux constraint

Here we will give an illustrative example which consists in the coupling of a two-dimensional and a one-dimensional model. Let us consider the domain illustrated in Fig. 8. In $\Omega_{1}$ we define for

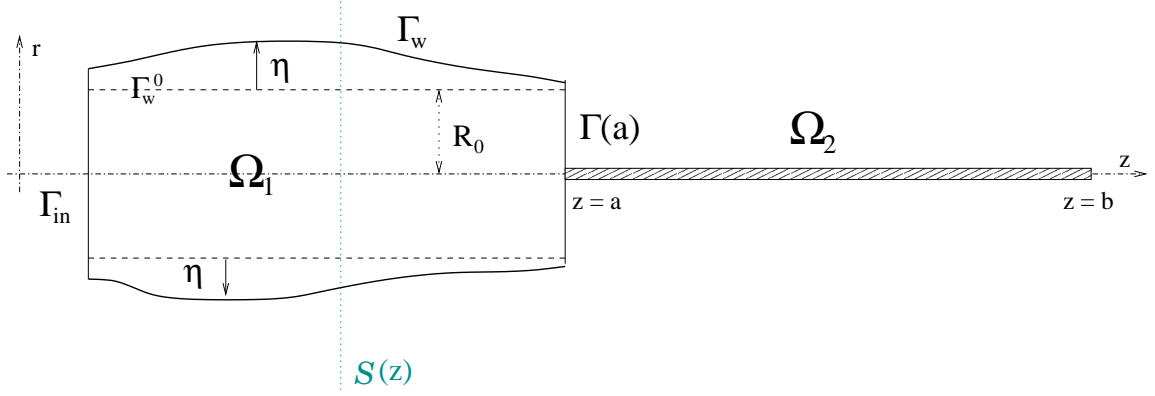

Figure 8: Coupled 2D/1D problem. On the left, the $2 \mathrm{D}$ model $\left(\Omega_{1}\right)$, where $\Gamma_{w}$ represents the arterial wall and $\eta$ the wall displacement with respect to the reference configuration $\Gamma_{w}^{0}$. On the right, the 1D model $\left(\Omega_{2}\right)$ defined on the interval $a \leq z \leq b$.

$t>0$ the fluid-structure model as

$$
\begin{cases}\rho \partial_{t} \mathbf{u}+\rho \mathbf{u} \cdot \nabla \mathbf{u}+\nabla p-\mu \triangle \mathbf{u}=0 & \text { in } \Omega_{1} \\ \operatorname{div} \mathbf{u}=0 & \text { in } \Omega_{1} \\ \rho_{w} h \frac{\partial^{2} \eta}{\partial t^{2}}-k G h \frac{\partial^{2} \eta}{\partial z^{2}}+\frac{E h}{1-\nu^{2}} \frac{\eta}{R_{0}^{2}}-\gamma \frac{\partial^{3} \eta}{\partial z^{2} \partial t}=f(t, z) & \text { on } \Gamma_{w}^{0} \\ \partial_{t} \eta \mathbf{e}_{r}=\mathbf{u} & \text { on } \Gamma_{w} \\ f(t, z)=\left(p \mathbf{n}-\mu \frac{\partial \mathbf{u}}{\partial \mathbf{n}}\right) \cdot \mathbf{e}_{r} \sqrt{1+\left(\frac{\partial \eta}{\partial z}\right)^{2}} & \text { on } \Gamma_{w}^{0}\end{cases}
$$

where the unknowns are the fluid velocity $\mathbf{u}$, the fluid pressure $p$ and the wall displacement $\eta$. Here, $\rho$ is the fluid density, $h$ the vessel wall thickness, $E$ the Young modulus, $G$ the Timoshenko factor and $\rho_{w}$ the wall density. At $t=0$ initial conditions $\mathbf{u}_{0}, \eta_{0}, \dot{\eta}_{0}$ are provided for the velocity, displacement and displacement rate, respectively. We refer to [3] for a more detailed description and analysis of this problem. In $\Omega_{2}$ we consider the following one dimensional problem for the velocity flux $Q$ and the vessel section area $A$ 


$$
\begin{cases}\frac{\partial A}{\partial t}+\frac{\partial Q}{\partial z}=0, & a<z<b, t>0 \\ \frac{\partial Q}{\partial t}+\frac{\partial}{\partial z}\left(\alpha \frac{Q^{2}}{A}\right)+\frac{A}{\rho} \frac{\partial \bar{p}}{\partial z}+K_{R} \frac{Q}{A}=0, & a<z<b, t>0 .\end{cases}
$$

supplied with the algebraic relation $\bar{p}=\beta\left(A-A_{0}\right), A_{0}$ being the reference area $A_{0}=2 R_{0}$. The system is supplemented by initial conditions for $A$ and $Q$ at $t=0$. This one dimensional reduced model is basically derived from (38), integrating the Navier-Stokes equations over each axial section $\mathcal{S}(z)$ and adopting a simplified version of the equation for the wall dynamics. $A$ is the measure of $\mathcal{S}(z)$, the velocity flux is given by $Q(z)=\int_{\mathcal{S}(z)} u_{z} d r$ and $\bar{p}(z)=\left(\int_{\mathcal{S}(z)} p d r\right) / A(z)$; see [5] for more details.

System (38) has been discretised in space using P1-isoP2 finite elements for the fluid and P1 elements for the structure. For time discretisation, we have adopted an ALE formulation to account for the domain movement with an implicit Euler discretisation for the fluid equations and a Newmark scheme for the structure. On the other hand, system (39), which is hyperbolic, has been discretised using a second-order Taylor-Galerkin scheme with a characteristic treatment of the boundary.

At each time step $t^{n}$, we look for a solution of (38) and (39) which satisfies at $\Gamma(a)$ the coupling conditions

$$
\operatorname{meas}\left(\Gamma^{n}(a)\right)=A^{n}(a), \quad \int_{\Gamma^{n}(a)} u_{z}^{n}=Q^{n}(a), \quad \frac{1}{\operatorname{meas}\left(\Gamma^{n}(a)\right)} \int_{\Gamma(a)} p^{n}=\bar{p}^{n}(a)
$$

We have solved iteratively at each time step the two sub-problems in $\Omega_{1}$ and $\Omega_{2}$. Given the approximate solution $\mathbf{u}^{n}, p^{n}, \eta^{n}, Q^{n}$ and $A^{n}$ of the coupled problem at time $t=t^{n}$, we look for the solution $\mathbf{u}^{n+1}, p^{n+1}, \eta^{n+1}, Q^{n+1}$ and $A^{n+1}$ using the following iterative algorithm : we set $\mathbf{u}_{(0)}=\mathbf{u}^{n}, p_{(0)}=p^{n}$, and $\eta_{(0)}=\eta^{n}$ and for $k=0,1, \ldots$

1. we solve the $1 \mathrm{D}$ model (39) imposing at $z=a$

$$
A_{(k+1)}(a)=A_{0}+\frac{1}{\beta \operatorname{meas}\left(\Gamma_{(k)}(a)\right)} \int_{\Gamma(a)} p_{(k)}
$$

and at $z=b$ absorbing boundary conditions based on characteristic analysis. We obtain $Q_{(k+1)}$ and $A_{(k+1)}$ in $\Omega_{2}$;

2. we solve then the $2 \mathrm{D}$ problem imposing on $\Gamma(a)$ for the Navier-Stokes equations the defective condition

$$
\int_{\Gamma(a)} \mathbf{u}_{(k+1)} \cdot \mathbf{e}_{z}=Q_{(k+1)}(a)
$$

and for the structure at $z=a$

$$
\eta_{(k+1)}(a)=\frac{1}{2} A_{(k+1)}(a)-R_{0}
$$

We obtain $\mathbf{u}_{(k+1)}, p_{(k+1)}, \eta_{(k+1)}$ in $\Omega_{1}$.

We iterate until the coupling conditions are satisfied within a fixed tolerance and we finally set the solution at time $t^{n+1}$ equal to the converged value. We may eventually add a relaxation step on the variable $A_{(k)}(a)$.

We observe that in step 2. of this algorithm we have to solve Navier-Stokes equations with flux boundary conditions on $\Gamma(a)$. A different algorithm for the same coupled 2D/1D problem, which allows to impose a mean pressure condition on $\Gamma(a)$, has been proposed and analysed in [3]. 

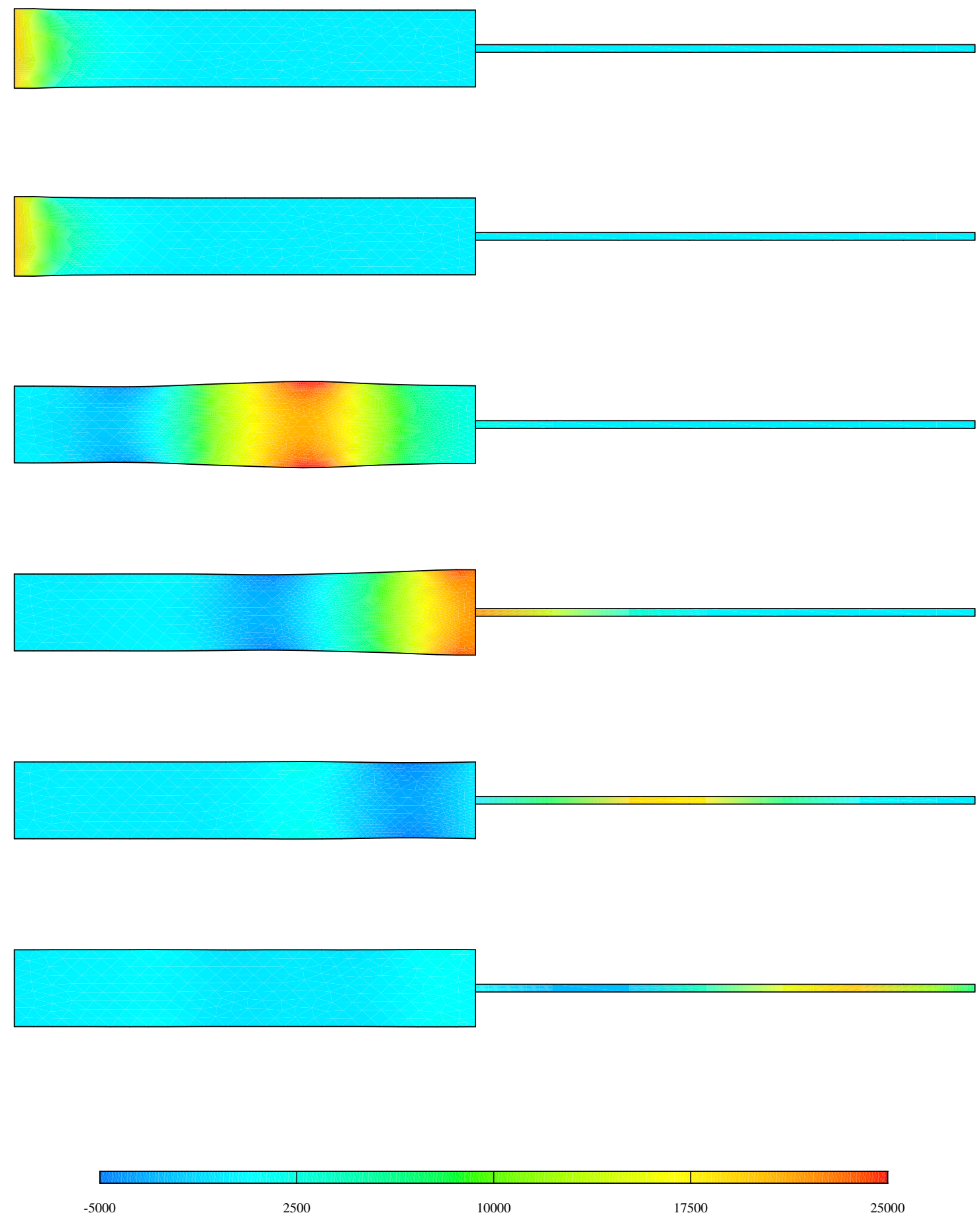

Figure 9: Coupling 2D simulation with the 1D reduced model; pressure distribution every $5 \mathrm{~ms}$, starting from $t=1 \mathrm{~ms}$.

We present here the numerical results relative to the following test case: we have considered a fluid initially at rest and we have imposed a pressure of $15 \mathrm{mmHg}\left(2 \cdot 10^{4}\right.$ dynes $\left./ \mathrm{cm}^{2}\right)$ at the inlet $\left(\Gamma_{i n}\right)$ for 0.005 seconds. For the fluid we have taken $\mu=0.035$ poise and $\rho=1 \mathrm{~g} / \mathrm{cm}^{3}$, while for 
the structure we have $E=0.75 \cdot 10^{6}$ dynes $/ \mathrm{cm}^{2}, \nu=0.5, \rho_{w}=1.1 \mathrm{~g} / \mathrm{cm}^{3}$ and $h=0.1 \mathrm{~cm}$. Figure 9 shows the fluid pressure and the domain deformation at different times. We may note how the "pressure wave" crosses the interface between the two models with little spurious reflections.

\section{Conclusions}

In this work we have considered defective boundary conditions for the Navier-Stokes equations. In particular, we have addressed the case where one wants to impose the mass flux on a measurable subset of the domain boundary. We have proposed a formulation based on a Lagrange multiplier technique and we have shown that it is well posed for the Stokes and the linearized Navier-Stokes equations. Moreover, we have considered some numerical algorithms to effectively solve the mixed problem thus obtained. Finally, we have presented two applications in which the technique may be advantageously used and we have shown some numerical results illustrating its effectiveness.

\section{Acknowledgements}

The work has been partially supported by the Swiss National Science Foundation, project N. 2154139.98, by MURST Cofin. 1998 "Advanced Numerical Methods for Scientific Computing" and by the special project of the Politecnico di Milano "Multiscale Computing in Biofluidynamics".

\section{References}

[1] C. Bègue, C. Conca, F. Murat, and O. Pironneau. Les équations de Stokes et de Navier-Stokes avec des conditions aux limites sur la pression. In Nonlinear partial differential equations and their applications. Collège de France Seminar, Vol. IX (Paris, 1985-1986), pages 179-264. Longman Sci. Tech., Harlow, 1988.

[2] F. Brezzi and M. Fortin. Mixed and Hybrid Finite Elements. SSCM n. 5, Springer-Verlag, 1991.

[3] L. Formaggia, J.-F. Gerbeau, F. Nobile, and A. Quarteroni. On the coupling of 3D and 1D Navier-Stokes equations for flow problems in compliant vessels. Technical Report 3862, INRIA, 2000. to appear on Comp. Methods in Appl. Mech. Engng.

[4] L. Formaggia and F. Nobile. A stability analysis for the Arbitrary Lagrangian Eulerian formulation with finite elements. East-West J. Num. Math., 7:105-132, 1999.

[5] L. Formaggia, F. Nobile, A. Quarteroni, and A. Veneziani. Multiscale Modelling of the Circulatory System: a preliminary analysis. Comp. Vis. Science, 2:75-83, 1999.

[6] V. Girault and P.R. Raviart. Navier-Stokes Equations: Theory and Numerical Analysis. Springer Series in Computational Mathematics (5), 1986.

[7] J.G. Heywood, R. Rannacher, and S. Turek. Artificial boundaries and flux and pressure conditions for the incompressible Navier-Stokes equations. International Journal for Numerical Methods in Fluids, 22:325-352, 1996.

[8] D.A. McDonald. Blood Flow in Arteries. Edward Arnold Ltd. - London, 1990. Third edition edited by W.W. Nichols and M.F. O' Rourke.

[9] F.C. Otto and G. Lube. A non-overlapping domain decomposition method for the Oseen equations. Math. Models Meth. Appl. Sci., 8(6):1091-1117, 1998.

[10] B. Perot. An analysis of the fractional step method. J. Comp. Phys., 108:51-58, 1993. 
[11] A. Quarteroni, F. Saleri, and A. Veneziani. The Yosida method for the numerical approximation of Navier-Stokes equations. J. Pure Appl. Math., 78:473-503, 1999.

[12] A. Quarteroni, M. Tuveri, and A. Veneziani. Computational Vascular Fluid Dynamics: Problems, Models and Methods. Comp. Vis. Science, 2:163-197, 2000.

[13] A. Quarteroni and A. Valli. Numerical Approximation of Partial Differential Equations. Springer Verlag Series in Computational Mathematics n.23, 1994.

[14] Alfio Quarteroni, Fausto Saleri, and Alessandro Veneziani. Factorization methods for the numerical approximation of Navier-Stokes equations. Comput. Methods Appl. Mech. Engrg., 188(1-3):505-526, 2000.

[15] R. Temam. Navier-Stokes equations. Theory and numerical analysis. North-Holland, Amsterdam, 1984. 3rd ed. 


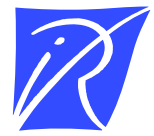

Unité de recherche INRIA Rocquencourt Domaine de Voluceau - Rocquencourt - BP 105 - 78153 Le Chesnay Cedex (France)

Unité de recherche INRIA Lorraine : LORIA, Technopôle de Nancy-Brabois - Campus scientifique 615, rue du Jardin Botanique - BP 101 - 54602 Villers-lès-Nancy Cedex (France)

Unité de recherche INRIA Rennes : IRISA, Campus universitaire de Beaulieu - 35042 Rennes Cedex (France)

Unité de recherche INRIA Rhône-Alpes : 655, avenue de l'Europe - 38330 Montbonnot-St-Martin (France)

Unité de recherche INRIA Sophia Antipolis : 2004, route des Lucioles - BP 93 - 06902 Sophia Antipolis Cedex (France)

INRIA - Domaine de Voluceau - Rocquencourt, BP 105 - 78153 Le Chesnay Cedex (France)

http://www.inria.fr

ISSN 0249-6399 